\title{
DOMAIN-INDEPENDENT UPPER BOUNDS FOR EIGENVALUES OF ELLIPTIC OPERATORS
}

\author{
STEPHEN M. HOOK
}

Abstract. Let $\Omega \subseteq \mathbb{R}^{m}$ be a bounded open set, $\partial \Omega$ its boundary and $\Delta$ the Laplacian on $\mathbb{R}^{m}$. Consider the elliptic differential equation:

$$
-\Delta u=\lambda u \text { in } \Omega ; \quad u=0 \text { on } \partial \Omega .
$$

It is known that the eigenvalues, $\lambda_{i}$, of (1) satisfy

$$
\sum_{i=1}^{n} \frac{\lambda_{i}}{\lambda_{n+1}-\lambda_{i}} \geq \frac{m n}{4}
$$

provided that $\lambda_{n+1}>\lambda_{n}$.

In this paper we abstract the method used by Hile and Protter [2] to establish (2) and apply the method to a variety of second-order elliptic problems, in particular, to all constant coefficient problems. We then consider a variety of higher-order problems and establish an extension of (2) for problem (1) where the Laplacian is replaced by a more general operator in a Hilbert space.

\section{INTRODUCTION}

Let $\Delta$ be the Laplace operator in $\mathbb{R}^{m}$ and $\Omega \subseteq \mathbb{R}^{m}$ a bounded region and $\partial \Omega$ its boundary in which the eigenvalue problem

$$
-\Delta u=\lambda u \quad \text { in } \Omega ; \quad u=0 \quad \text { on } \partial \Omega
$$

has discrete spectrum $\lambda_{1} \leq \lambda_{2} \leq \cdots \leq \lambda_{n}<\lambda_{n+1} \leq \cdots$. Then the eigenvalues of (1.1) satisfy

$$
\sum_{i=1}^{n} \frac{\lambda_{i}}{\lambda_{n+1}-\lambda_{i}} \geq \frac{m n}{4} .
$$

The result (1.2) was established by Hile and Protter [2] and is a generalization of

$$
\lambda_{n+1} \leq \lambda_{n}+\frac{2}{n} \sum_{i=1}^{n} \lambda_{i}
$$

for $m=2$. Result (1.3) was established by Payne, Polya, and Weinberger [8]. Inequality (1.3) follows from (1.2) since the summand in (1.2) is bounded above

Received by the editors July 12, 1988 .

1980 Mathematics Subject Classification (1985 Revision). Primary 35J35, 35P15; Secondary $35 \mathrm{G} 15,35 \mathrm{~J} 40,35 \mathrm{~J} 30$. 
by $\lambda_{i} /\left(\lambda_{n+1}-\lambda_{n}\right)$ for each $i$. The interesting thing about (1.2) is that it leads to an upper bound on the $(n+1)$ th eigenvalue of the Dirichlet problem, $(1.1)$, in terms of the lower eigenvalues which is domain-independent. That is, there is no explicit dependence on the size or shape of the domain $\Omega$ in (1.2).

More recently Hile and Yeh [3] and Chen [1] considered polyharmonic operators of the form

$$
(-\Delta)^{l} u=\lambda u \quad \text { in } \Omega ; \quad u=\frac{\partial u}{\partial n}=\cdots=\frac{\partial^{l-1} u}{\partial n^{l-1}}=0 \quad \text { on } \partial \Omega,
$$

where $\partial / \partial n$ denotes the outward-pointing normal derivative on $\partial \Omega$. Hile and Yeh [3] considered problem (1.4) with $l=2$ and Chen [1] considered certain other even positive integers $l$. The result of [3] and [1] is that

$$
\left(\sum_{i=1}^{n} \frac{\lambda_{i}^{1 / l}}{\lambda_{n+1}-\lambda_{i}}\right)\left(\sum_{i=1}^{l} \lambda_{i}\right)^{(l-1) / l} \geq \frac{m^{2} n^{2 l-1}}{4 l(2 l+m-2)} .
$$

Again (1.5) has no explicit dependence on the domain $\Omega$.

In this paper we abstract the method (Theorem 1) of [2] to a Hilbert space. After establishing the abstract generalization of this method in $\S 2$, we establish the result (1.2) as a strict inequality or one which is similar for a variety of second-order elliptic differential equations in one dependent variable. We establish (1.2) as a strict inequality for

$$
\begin{gathered}
-e^{\mathbf{w} \cdot \mathbf{x}} \operatorname{div}\left(M^{2} e^{-\mathbf{w} \cdot \mathbf{x}} \operatorname{grad} u\right)-\frac{1}{4}\|M \mathbf{w}\|^{2} u=\lambda u \quad \text { in } \Omega ; \\
u=0 \quad \text { on } \partial \Omega,
\end{gathered}
$$

where $M$ is a positive definite real symmetric matrix of constants, grad is the gradient, div is the divergence, $\mathbf{w}$ is a constant $\mathbf{w} \in \mathbb{R}^{m}, \mathbf{w} \cdot \mathbf{x}$ is the dot problem in $\mathbb{R}^{m}$ and $\|\cdot\|$ is the $\mathbb{R}^{m}$-Euclidean norm. Every (real) constant coefficient second-order elliptic problem with the boundary conditions of (1.6) can be put into the form (1.6), in particular those with first-order terms.

Results similar to (1.2) hold for

$$
-\Delta u-2 i \mathbf{w} \cdot \operatorname{grad} u=\lambda u \quad \text { in } \Omega ; \quad u=0 \text { on } \partial \Omega
$$

in which $i^{2}=-1$ (not to be confused with the index) and $\mathbf{w} \in \mathbb{R}^{m}$ is a constant. For problem (1.7), if the eigenvalues are discrete and $\lambda_{n+1}>\lambda_{n}$ then

$$
\sum_{i=1}^{n} \frac{\lambda_{i}-\|\mathbf{w}\|^{2}}{\lambda_{n+1}-\lambda_{i}}>\frac{m n}{4}
$$

holds.

Finally the problem

$$
-\Delta u+\rho(x) u=\lambda u \quad \text { in } \Omega ; \quad u=0 \text { on } \partial \Omega
$$

Satisfies (1.2), where $\rho(x) \geq 0$ in $\Omega$, and for the Sturm-Liouville problem

$$
-\left((\rho(x))^{2} u^{\prime}\right)^{\prime}+g(x) u=\lambda u \quad \text { in } I=(\alpha, \beta),
$$




$$
u(\alpha)=u(\beta)=0,
$$

where $\rho(x)>0$ on $I$, the eigenvalues obey

$$
\sum_{i=1}^{n} \frac{\lambda_{i}-M}{\lambda_{n+1}-\lambda_{i}} \geq \frac{n}{4}
$$

where $M$ is any constant so that $M \leq g-\frac{1}{4}\left(\left(\rho^{\prime}\right)^{2}-\left(\rho^{2}\right)^{\prime \prime}\right)$ in $I$ and the "prime" notation denotes the derivative.

The abstraction in Theorem 1 along with another theorem of this author [4] or [5] is used to establish an improvement of (1.5) for the problem

$$
p(-\Delta) u=\lambda u \quad \text { in } \Omega, \quad u=\frac{\partial u}{\partial n}=\cdots=\frac{\partial^{l-1} u}{\partial n^{l-1}}=0 \quad \text { on } \partial \Omega,
$$

where $p$ is a monic polynomial (leading coefficient is unity) of degree $l>0$. In particular if $p$ has nonnegative coefficients and $p(0)=0$ then

$$
\left(\sum_{i=1}^{n} \frac{\lambda_{i}^{1 / l}}{\lambda_{n+1}-\lambda_{i}}\right)\left(\sum_{i=1}^{n} \frac{m}{2} p^{\prime}\left(\lambda_{i}^{1 / l}\right)+\lambda_{i}^{1 / l} p^{\prime \prime}\left(\lambda_{i}^{1 / l}\right)\right)>\frac{m^{2} n^{2}}{8} .
$$

We also note that the result holds for polynomials in more general constant coefficient second-order equations and that the eigenvalues of some higher-order problems other than ones in the form of (1.12) satisfy similar inequalities.

Finally we consider the system of equations for an elastic medium

$$
-\Delta \mathbf{u}-\alpha \operatorname{grad}(\operatorname{div}(\mathbf{u}))=\Lambda \mathbf{u} \quad \text { in } \Omega, \quad \mathbf{u}=\mathbf{0} \quad \text { on } \partial \Omega .
$$

Equation (1.14) is a system of $m$ partial differential equations. The symbol $\Delta$ is the operator which takes the Laplacian of each component of a vector field $\mathbf{u}$, and $\alpha$ is a constant. We establish that if $\Omega \subseteq \mathbb{R}^{m}$ is bounded and (1.14) has discrete eigenvalues $\Lambda_{1} \leq \Lambda_{2} \leq \cdots \leq \Lambda_{n}<\Lambda_{n+1} \leq \cdots$ and $\alpha \geq 0$ then

$$
\sum_{i=1}^{n} \frac{\Lambda_{i}}{\Lambda_{n+1}-\Lambda_{i}} \geq \frac{m^{2} n}{4(m+\alpha)}
$$

holds.

As noted before, none of the results of this paper have any explicit dependence on the domain, $\Omega$, except for the dimension $m$. We begin $\S 2$ with the abstraction of the method of [2].

\section{AN ABSTRACT THEOREM}

Our results depend on a rather complicated-looking theorem. It is given abstractly with applications in subsequent sections. For linear operators $L$ and $M$ we define the commutator, $[L, M]$, by $[L, M] u=L M u-M L u$. We have the following theorem.

Theorem 1. Let $A: D \subseteq \mathscr{H} \rightarrow \mathscr{H}$ be a selfadjoint operator in a real or complex Hilbert space $\mathscr{H}$, with inner product $\langle\cdot, \cdot\rangle$, which is semibounded below with 
discrete spectrum $\lambda_{1} \leq \lambda_{2} \leq \cdots$. Let $\left\{T_{j}: D \rightarrow \mathscr{H}\right\}_{j=1}^{M}$ be any collection of skew-symmetric operators and $\left\{B_{j}: T_{j}(D) \rightarrow \mathscr{H}\right\}_{j=1}^{M}$ any collection of symmetric operators which leave $D$ invariant. Denote the normalized eigenvectors of $A$ by $\left\{u_{i}\right\}_{i=1}^{\infty}$, where $u_{i}$ corresponds to $\lambda_{i}$. Suppose that $\lambda_{n+1}>\lambda_{n}$. If for some $i$ and $j, T_{j} u_{i} \neq 0$ and if there is $a \gamma>0$ and $\beta>\lambda_{n}$ so that

$$
\begin{gathered}
\gamma \sum_{i=1}^{n} \sum_{j=1}^{M}\left\langle\left[T_{j}, B_{j}\right] u_{i}, u_{i}\right\rangle+\gamma^{2} \sum_{i=1}^{n} \sum_{j=1}^{M} \frac{1}{\beta-\lambda_{i}}\left\langle T_{j} u_{i}, T_{j} u_{i}\right\rangle \\
=\frac{1}{2} \sum_{i=1}^{n} \sum_{j=1}^{M}\left\langle\left[\left[A, B_{j}\right], B_{j}\right] u_{i}, u_{i}\right\rangle
\end{gathered}
$$

then $\beta \geq \lambda_{n+1}$. Moreover if $T_{j} u_{i} \notin D$ for all $i=1,2, \ldots, n$ and $j=$ $1,2, \ldots, M$ we have $\beta>\lambda_{n+1}$.

Proof. We construct a collection of test functions, $\phi_{i j}$, which are orthogonal to each of the first $n$ eigenvectors and apply well-known minimizing properties of $\lambda_{n+1}$. For each $i=1,2, \ldots, n$ and $j=1,2, \ldots, M$, define

$$
\phi_{i j}=B_{j} u_{i}-\sum_{k=1}^{n} a_{j i k} u_{k}
$$

where

$$
a_{j i k}=\left\langle B_{j} u_{i}, u_{k}\right\rangle .
$$

From (2.2) and (2.3) we see that

$$
\left\langle\phi_{i j}, u_{k}\right\rangle=0 \text { for all } i, k=1,2, \ldots, n \text { and } j=1,2, \ldots, M .
$$

Then for each $i, j$ we have

$$
\lambda_{n+1}\left\langle\phi_{i j}, \phi_{i j}\right\rangle \leq\left\langle A \phi_{i j}, \phi_{i j}\right\rangle
$$

since each $\phi_{i j}$ is orthogonal to each of the first $n$ eigenvectors of $A$ by (2.4).

Next we work on the right-hand side of (2.5). By the definition of the $\phi_{i j}$ and (2.4) we have

$$
\left\langle A \phi_{i j}, \phi_{i j}\right\rangle=\left\langle A B_{j} u_{i}, \phi_{i j}\right\rangle .
$$

We apply the definition of the commutator and the fact that $A u_{i}=\lambda_{i} u_{i}$ to the right-hand side of (2.6) we obtain

$$
\begin{aligned}
\left\langle A \phi_{i j}, \phi_{i j}\right\rangle & =\left\langle B_{j} A u_{i}, \phi_{i j}\right\rangle+\left\langle\left[A, B_{j}\right] u_{i}, \phi_{i j}\right\rangle \\
& =\lambda_{i}\left\langle B_{j} u_{i}, \phi_{i j}\right\rangle+\left\langle\left[A, B_{j}\right] u_{i}, \phi_{i j}\right\rangle .
\end{aligned}
$$

Since $\left\langle B_{j} u_{i}, \phi_{i j}\right\rangle=\left\langle\phi_{i j}, \phi_{i j}\right\rangle$ by definition of $\phi_{i j}$ and the orthogonality condition, (2.4), we can rewrite (2.7) as

$$
\left\langle A \phi_{i j}, \phi_{i j}\right\rangle=\lambda_{i}\left\langle\phi_{i j}, \phi_{i j}\right\rangle+\left\langle\left[A, B_{j}\right] u_{i}, \phi_{i j}\right\rangle .
$$


Substitution of the definition of $\phi_{i j}$ into the rightmost term of (2.8), substitution of the result into the right-hand side of (2.5) and summing this over all $i$ yields

$$
\begin{aligned}
\lambda_{n+1} \sum_{i=1}^{n}\left\langle\phi_{i j}, \phi_{i j}\right\rangle \leq & \sum_{i=1}^{n} \lambda_{i}\left\langle\phi_{i j}, \phi_{i j}\right\rangle+\sum_{i=1}^{n}\left\langle\left[A, B_{j}\right] u_{i}, B_{j} u_{i}\right\rangle \\
& -\sum_{i=1}^{n} \sum_{k=1}^{n}\left\langle\left[A, B_{j}\right] u_{i}, a_{j i k} u_{k}\right\rangle .
\end{aligned}
$$

The right-hand side of (2.9) is real so (2.9) still holds if we take the real part of the right-hand side. (Notice that in a real Hilbert space the operation of obtaining the real part still makes sense!) As it is, the real part of the double sum is zero since

$$
\begin{aligned}
\operatorname{Re} & \sum_{k, i=1}^{n}\left\langle\left[A, B_{j}\right] u_{i}, a_{j i k} u_{k}\right\rangle \\
& =\frac{1}{2}\left[\sum_{i, k=1}^{n}\left\langle\left[A, B_{j}\right] u_{i}, a_{j i k} u_{k}\right\rangle+\overline{\sum_{i, k=1}^{n}\left\langle\left[A, B_{j}\right] u_{i}, a_{j i k} u_{k}\right\rangle}\right] \\
& =\frac{1}{2}\left[\sum_{i, k=1}^{n}\left\langle\left[A, B_{j}\right] u_{i}, a_{j i k} u_{k}\right\rangle+\sum_{i, k=1}^{n}\left\langle a_{j i k} u_{k},\left[A, B_{j}\right] u_{i}\right\rangle\right] \\
& =\frac{1}{2}\left[\sum_{i, k=1}^{n}\left\langle\left[A, B_{j}\right] u_{i}, a_{j i k} u_{k}\right\rangle+\sum_{i, k=1}^{n}\left\langle-\left[A, B_{j}\right] u_{k}, a_{j k i} u_{i}\right\rangle\right] .
\end{aligned}
$$

The last step is justified since the adjoint $\left[A, B_{j}\right]^{*}=-\left[A, B_{j}\right]$ whenever $A$ and $B_{j}$ are symmetric and the fact that $\overline{a_{j i k}}=a_{j k i}$ for all $i, k, j$. Switching $i$ and $k$ in either double sum in the right-hand side of (2.10) establishes that the real part of the double sum is (2.9) is, indeed, zero.

After taking real parts in (2.9) we have

$$
\lambda_{n+1} \sum_{i=1}^{n}\left\langle\phi_{i j}, \phi_{i j}\right\rangle \leq \sum_{i=1}^{n} \lambda_{i}\left\langle\phi_{i j}, \phi_{i j}\right\rangle+\frac{1}{2} \sum_{i=1}^{n}\left(\left\langle\left[A, B_{j}\right] u_{i}, B_{j} u_{i}\right\rangle+\left\langle B_{j} u_{i},\left[A, B_{j}\right] u_{i}\right\rangle\right) \text {. }
$$

The symmetry of $B_{j}$, skew symmetry of $\left[A, B_{j}\right]$ and the definition of the commutator are used to establish that

$$
\frac{1}{2} \sum_{i=1}^{n}\left(\left\langle\left[A, B_{j}\right] u_{i}, B_{j} u_{i}\right\rangle+\left\langle B_{j} u_{i},\left[A, B_{j}\right] u_{i}\right\rangle\right)=-\frac{1}{2} \sum_{i=1}^{n}\left\langle\left[\left[A, B_{j}\right], B_{j}\right] u_{i}, u_{i}\right\rangle .
$$

Thus (2.11) becomes

$$
\lambda_{n+1} \sum_{i=1}^{n}\left\langle\phi_{i j}, \phi_{i j}\right\rangle \leq \sum_{i=1}^{n} \lambda_{i}\left\langle\phi_{i j}, \phi_{i j}\right\rangle-\frac{1}{2} \sum_{i=1}^{n}\left\langle\left[\left[A, B_{j}\right], B_{j}\right] u_{i}, u_{i}\right\rangle .
$$


Next let $\beta>\lambda_{n}$ be a real constant and subtract $\beta \sum_{i=1}^{n}\left\langle\phi_{i j}, \phi_{i j}\right\rangle$ from both sides of (2.13) and sum the result over all $j$. This yields

$$
\begin{aligned}
& \left(\lambda_{n+1}-\beta\right) \sum_{i=1}^{n} \sum_{j=1}^{M}\left\langle\phi_{i j}, \phi_{i j}\right\rangle \\
& \quad \leq \sum_{i=1}^{n} \sum_{j=1}^{M}\left(\lambda_{i}-\beta\right)\left\langle\phi_{i j}, \phi_{i j}\right\rangle-\frac{1}{2} \sum_{i=1}^{n} \sum_{j=1}^{M}\left\langle\left[\left[A, B_{j}\right], B_{j}\right] u_{i}, u_{i}\right\rangle .
\end{aligned}
$$

At this point we want to find an upper bound for $\sum_{i=1}^{n} \sum_{j=1}^{M}\left(\lambda_{i}-\beta\right)\left\langle\phi_{i j}, \phi_{i j}\right\rangle$ expressed in terms of the $u_{i}$ and certain operators. To do this, recall that in any Hilbert space if $\delta>0$ we have

$$
\operatorname{Re}\langle u, v\rangle \leq \frac{\delta}{2}\langle u, u\rangle+\frac{1}{2 \delta}\langle v, v\rangle
$$

with strict inequality except when $\delta u=v$. In particular, if $u$ and $v$ are linearly independent, inequality $(2.15)$ is strict. For each $j$ we have

$$
\begin{aligned}
\sum_{i=1}^{n} \operatorname{Re}\left\langle\phi_{i j}, T_{j} u_{i}\right\rangle= & \frac{1}{2} \sum_{i=1}^{n}\left(\left\langle B_{j} u_{i}, T_{j} u_{i}\right\rangle+\left\langle T_{j} u_{i}, B_{j} u_{i}\right\rangle\right) \\
& -\sum_{i, k=1}^{n}\left(\left\langle a_{j i k} u_{k}, T_{j} u_{i}\right\rangle+\left\langle T_{j} u_{i}, a_{j i k} u_{k}\right\rangle\right) \\
= & \frac{1}{2} \sum_{i=1}^{n}\left\langle\left[B_{j}, T_{j}\right] u_{i}, u_{i}\right\rangle-\operatorname{Re} \sum_{i=1}^{n} \sum_{k=1}^{n}\left\langle a_{j i k} u_{k}, T_{j} u_{i}\right\rangle .
\end{aligned}
$$

Since $a_{j i k}=\overline{a_{j k i}}$ for each $j, k$ and $i$ and $T_{j}$ is skew-symmetric, the double sum in (2.16) must be zero as it simply represents the sum of all the entries of a skew-symmetric matrix. Thus we have

$$
\operatorname{Re} \sum_{i=1}^{n}\left\langle\phi_{i j}, T_{j} u_{i}\right\rangle=\frac{1}{2} \sum_{i=1}^{n}\left\langle\left[B_{j}, T_{j}\right] u_{i}, u_{i}\right\rangle .
$$

Application of (2.15) to the left-hand side of (2.17) with $\delta=\left(\beta-\lambda_{i}\right) / \gamma$ yields (2.18)

$$
\frac{1}{2} \sum_{i=1}^{n}\left\langle\left[B_{j}, T_{j}\right] u_{i}, u_{i}\right\rangle \leq \frac{1}{2 \gamma} \sum_{i=1}^{n}\left(\beta-\lambda_{i}\right)\left\langle\phi_{i j}, \phi_{i j}\right\rangle+\frac{\gamma}{2} \sum_{i=1}^{n} \frac{1}{\beta-\lambda_{i}}\left\langle T_{j} u_{i}, T_{j} u_{i}\right\rangle .
$$

Inequality (2.18) holds for all positive $\gamma$. Rearrangement of (2.18) and summing over $j$ yields the desired upper bound:

$$
\begin{aligned}
& \sum_{j=1}^{M} \sum_{i=1}^{n}\left(\lambda_{i}-\beta\right)\left\langle\phi_{i j}, \phi_{i j}\right\rangle \\
& \quad \leq-\gamma \sum_{j=1}^{M} \sum_{i=1}^{n}\left\langle\left[B_{j}, T_{j}\right] u_{i}, u_{i}\right\rangle+\gamma^{2} \sum_{j=1}^{M} \sum_{i=1}^{n} \frac{1}{\beta-\lambda_{i}}\left\langle T_{j} u_{i}, T_{j} u_{i}\right\rangle .
\end{aligned}
$$


Substitution of (2.19) into (2.14) yields

$$
\begin{aligned}
\left(\lambda_{n+1}-\beta\right) \sum_{i=1}^{n} \sum_{j=1}^{M}\left\langle\phi_{i j}, \phi_{i j}\right\rangle \leq & -\gamma \sum_{j=1}^{M} \sum_{i=1}^{n}\left\langle\left[B_{j}, T_{j}\right] u_{i}, u_{i}\right\rangle \\
& +\gamma^{2} \sum_{j=1}^{M} \sum_{i=1}^{n} \frac{1}{\beta-\lambda_{i}}\left\langle T_{j} u_{i}, T_{j} u_{i}\right\rangle \\
& -\frac{1}{2} \sum_{i=1}^{n} \sum_{j=1}^{M}\left\langle\left[\left[A, B_{j}\right], B_{j}\right] u_{i}, u_{i}\right\rangle .
\end{aligned}
$$

The right-hand side of (2.20) depends on $\beta$ since we have assumed that $T_{j} u_{i} \neq$ 0 for some $i$ and $j$. So if we can choose $\beta>\lambda_{n}$ and $\gamma>0$ so that the righthand side of (2.20) is zero then a larger $\beta$ makes the right-hand side negative, holding $\gamma$ constant. In particular the left-hand side of (2.20) can be made negative, so the quantity $\sum_{j=1}^{M} \sum_{i=1}^{n}\left\langle\phi_{i j}, \phi_{i j}\right\rangle>0$. Thus when the right-hand side of (2.20) is equal to zero we must have $\lambda_{n+1}-\beta \leq 0$. In other words, $\beta \geq \lambda_{n+1}$. Now the right-hand side of (2.20) is equal to zero precisely when

$$
\begin{gathered}
\gamma \sum_{j=1}^{M} \sum_{i=1}^{n}\left\langle\left[T_{j}, B_{j}\right] u_{i}, u_{i}\right\rangle+\gamma^{2} \sum_{i=1}^{n} \frac{1}{\beta-\lambda_{i}} \sum_{j=1}^{M}\left\langle T_{j} u_{i}, T_{j} u_{i}\right\rangle \\
=\frac{1}{2} \sum_{i=1}^{n} \sum_{j=1}^{M}\left\langle\left[\left[A, B_{j}\right], B_{j}\right] u_{i}, u_{i}\right\rangle .
\end{gathered}
$$

Equalities (2.21) and (2.1) are the same, with the above discussion establishing the weak inequality.

Notice that the upper bound, (2.19), comes from (2.15) and (2.16). In particular if $\phi_{i j}$ and $T_{j} u_{i}$ are linearly independent for some $i$ and $j$ the upper bound, (2.19), is actually a strict inequality. We have argued above that for some $i$ and $j, \phi_{i j} \neq 0$. Under the additional assumption that $T_{j} u_{i} \notin D$ for any $i$ and $j, \phi_{i j}$ and $T_{j} u_{i}$ cannot be linearly dependent since $\phi_{i j} \in D$. Thus if $T_{j} u_{i} \notin D$ for all $i=1,2, \ldots, n$ and $j=1, \ldots, M$ we must have $\beta>\lambda_{n+1}$ whenever $\beta$ satisfies (2.1).

Theorem 1 is an abstraction of a method used first by Payne, Polya, and Weinberger [8], refined later by Hile and Protter [2] and effectively used again in [3], [1], and [4]. The result does not look too useful since we do not yet know how to choose the $T_{j}$ and $B_{j}$. Clearly though, we need to be able to choose the $B_{j}$ and $T_{j}$ so that the inner products can be calculated. As it is, we can often choose the $T_{j}$ and $B_{j}$ so that $\left[T_{j}, B_{j}\right] u=-u, \sum_{j=1}^{M}\left\langle T_{j} u, T_{j} u\right\rangle=\langle A u, u\rangle$ and $\left[\left[A, B_{j}\right], B_{j}\right] u=-2 u$ as follows.

Proposition 1. If $A, T_{j}$ and $B_{j}$ are as in Theorem 1, $A=-\sum_{j=1}^{M} T_{j}^{2}$ and if $\left[T_{j}, B_{i}\right] u=-\delta_{i j} u$ where $\delta_{i j}$ is the Kronecker symbol then

$$
\left[\left[A, B_{j}\right], B_{j}\right] u=-2 u \text {. }
$$


Proof.

$$
\begin{aligned}
{\left[A, B_{j}\right] u } & =-\sum_{k=1}^{M}\left[T_{k}^{2}, B_{j}\right] u=-\sum_{k=1}^{M}\left(T_{k}\left[T_{k}, B_{j}\right]+\left[T_{k}, B_{j}\right] T_{k}\right) u \\
& =-T_{j}\left[T_{j}, B_{j}\right] u-\left[T_{j}, B_{j}\right] T_{j} u=2 T_{j} u
\end{aligned}
$$

The sum collapses since $\left[T_{k}, B_{j}\right]=0$ if $k \neq j$. Thus $\left[\left[A, B_{j}\right], B_{j}\right] u=$ $\left[2 T_{j}, B_{j}\right] u=2\left[T_{j}, B_{j}\right] u=-2 u$.

Although it is not required, in our applications, $A$ is a differential operator, the $T_{j}$ are differential operators and the $B_{j}$ are multiplication operators. Proposition 1 effectively assures us that if $A=-\sum_{j=1}^{M} T_{j}^{2}$ where the $T_{j}$ are skew-symmetric and if a certain system of differential equations $\left(\left[T_{j}, B_{i}\right] u=-\delta_{i j} u\right)$ can be solved then we can expect to obtain a useful inequality from $(2.1)$.

\section{SECOND-ORDER DifFERENTIAL EQUATIONS}

We begin by considering the most general real constant coefficient elliptic problem with Dirichlet boundary conditions:

$$
\begin{gathered}
A u=-e^{\mathbf{w} \cdot \mathbf{x}} \operatorname{div}\left(M^{2} e^{-\mathbf{w} \cdot \mathbf{x}} \operatorname{grad} u\right)-\frac{1}{4}\|M \mathbf{w}\|^{2} u=\lambda u \quad \text { in } \Omega ; \\
u=0 \text { on } \partial \Omega,
\end{gathered}
$$

where $\Omega \subseteq \mathbb{R}^{m}$ is bounded, $M$ is a constant $m \times m$ (real) symmetric positive definite matrix and $\mathbf{w} \in \mathbb{R}^{m}$ is a constant vector. We apply Theorem 1 to problem (3.1) using the following lemmas.

Lemma 1. Let $D$ be a positive diagonal matrix and $U$ a real orthogonal matrix so that $M=U^{-1} D U$. For $j=1,2, \ldots, m$, the operators

$$
T_{j} u=\frac{1}{2} \mathbf{e}_{j} \cdot\left(e^{\mathbf{w} \cdot \mathbf{x}}\left(D U \operatorname{grad}\left(e^{-\mathbf{w} \cdot \mathbf{x}} u\right)\right)+D U \operatorname{grad}(u)\right)
$$

are skew-symmetric under the boundary conditions of (3.1) and inner product

$$
\langle u, v\rangle=\int_{\Omega} u v e^{-\mathbf{w} \cdot \mathbf{x}} .
$$

Moreover we have

$$
\sum_{j=1}^{m}\left\langle T_{j} u, T_{j} u\right\rangle=\langle A u, u\rangle
$$

and if $A u=\lambda u$ and $u \neq 0$ then $T_{j} u$ is not identically zero on $\partial \Omega$.

Proof. We need only show that $\left\langle T_{j} u, v\right\rangle=-\left\langle u, T_{j} v\right\rangle$, where $u$ and $v$ satisfy 
the boundary conditions of (3.1). But

$$
\begin{aligned}
\left\langle T_{j} u, v\right\rangle & =\frac{1}{2} \int_{\Omega} \mathbf{e}_{j} \cdot\left(D U \operatorname{grad}\left(e^{-\mathbf{w} \cdot \mathbf{x}} u\right) v+D U \operatorname{grad}(u) v e^{-\mathbf{w} \cdot \mathbf{x}}\right) \\
& =-\frac{1}{2} \int_{\Omega} \mathbf{e}_{j} \cdot\left(D U e^{-\mathbf{w} \cdot \mathbf{x}} u \operatorname{grad}(v)+D U u \operatorname{grad}\left(e^{-\mathbf{w} \cdot \mathbf{x}} v\right)\right) \\
& =-\frac{1}{2} \int_{\Omega} \mathbf{e}_{j} \cdot u\left(e^{\mathbf{w} \cdot \mathbf{x}}\left(D U \operatorname{grad}\left(e^{-\mathbf{w} \cdot \mathbf{x}} v\right)\right)+D U \operatorname{grad} v\right) e^{-\mathbf{w} \cdot \mathbf{x}} \\
& =-\left\langle u, T_{j} v\right\rangle .
\end{aligned}
$$

To establish (3.4), notice that (3.2) can be simplified to

$$
T_{j} u=\frac{1}{2} \mathbf{e}_{j} \cdot(2 D U \operatorname{grad} u-D U \mathbf{w} u) .
$$

Thus

$$
\sum_{j=1}^{m}\left\langle T_{j} u, T_{j} u\right\rangle=\frac{1}{4} \int_{\Omega}\|2 D U \operatorname{grad} u-D U \mathbf{w} u\|^{2} e^{-\mathbf{w} \cdot \mathbf{x}} .
$$

Using the norm-preserving property of $U$ and multiplying out the norm in (3.7) we see that

$$
\begin{aligned}
\frac{1}{4}\|2 D U \operatorname{grad} u-D U \mathbf{w} u\|^{2} & =\|D U \operatorname{grad} u\|^{2}-D U \operatorname{grad} u \cdot D U \mathbf{w} u+\frac{1}{4}\|D U \mathbf{w}\|^{2} u^{2} \\
& =\|M \operatorname{grad} u\|^{2}-M \operatorname{grad} u \cdot M \mathbf{w} u+\frac{1}{4}\|M \mathbf{w}\|^{2} u^{2} .
\end{aligned}
$$

Thus (3.7) becomes

$$
\begin{aligned}
\sum_{j=1}^{m}\left\langle T_{j} u, T_{j} u\right\rangle= & \int_{\Omega}\|M \operatorname{grad} u\|^{2} e^{-\mathbf{w} \cdot \mathbf{x}}-\int_{\Omega} M \operatorname{grad} u \cdot M \mathbf{w} u e^{-\mathbf{w} \cdot \mathbf{x}} \\
& +\frac{1}{4}\|M \mathbf{w}\|^{2} \int_{\Omega} u^{2} e^{-\mathbf{w} \cdot \mathbf{x}} .
\end{aligned}
$$

Notice that by the divergence theorem and boundary conditions we have

$$
\begin{aligned}
0 & =\int_{\Omega} \operatorname{div}\left(u M^{2} e^{-\mathbf{w} \cdot \mathbf{x}} \operatorname{grad} u\right) \\
& =\int_{\Omega} \operatorname{grad} u \cdot M^{2} \operatorname{grad}(u) e^{-\mathbf{w} \cdot \mathbf{x}}+\int_{\Omega} u \operatorname{div}\left(M^{2} e^{-\mathbf{w} \cdot \mathbf{x}} \operatorname{grad} u\right) \\
& =\int_{\Omega}\|M \operatorname{grad} u\|^{2} e^{-\mathbf{w} \cdot \mathbf{x}}+\int_{\Omega} \operatorname{div}\left(M^{2} e^{-\mathbf{w} \cdot \mathbf{x}} \operatorname{grad} u\right) u .
\end{aligned}
$$

Thus

$$
\int_{\Omega}\|M \operatorname{grad} u\|^{2} e^{-\mathbf{w} \cdot \mathbf{x}}=-\int_{\Omega} \operatorname{div}\left(M^{2} e^{-\mathbf{w} \cdot \mathbf{x}} \operatorname{grad} u\right) u .
$$

Also notice that when $u$ satisfies the boundary conditions of (3.1)

$$
\begin{aligned}
0 & =\int_{\Omega} \frac{1}{2} M^{2} \mathbf{w} \cdot \operatorname{grad}\left(u^{2} e^{-\mathbf{w} \cdot \mathbf{x}}\right) \\
& =\int_{\Omega} \frac{1}{2} M^{2} \mathbf{w} \cdot \operatorname{grad}(u) 2 u e^{-\mathbf{w} \cdot \mathbf{x}}-\frac{1}{2} \int_{\Omega} M^{2} \mathbf{w} \cdot \mathbf{w} u^{2} e^{-\mathbf{w} \cdot \mathbf{x}} .
\end{aligned}
$$


Upon rearranging (3.10) using the symmetry of $M$ we obtain

$$
\begin{aligned}
\int_{\Omega} M \operatorname{grad}(u) \cdot M \mathbf{w} u e^{-\mathbf{w} \cdot \mathbf{x}} & =\int_{\Omega} M^{2} \mathbf{w} \cdot \operatorname{grad}(u)\left(u e^{-\mathbf{w} \cdot \mathbf{x}}\right) \\
& =\frac{1}{2}\|M \mathbf{w}\|^{2} \int_{\Omega} u^{2} e^{-\mathbf{w} \cdot \mathbf{x}} .
\end{aligned}
$$

Placing the results of (3.9) and (3.11) into (3.8) shows that (3.4) holds.

Finally $T_{j} u$ is a linear combination of $u$ and a directional derivative of $u$. Thus the boundary conditions are such that $T_{j} u$ will satisfy them only if $\operatorname{grad} u=0$ on $\partial \Omega$. This is impossible for an eigenvector of $A$.

Lemma 2. There are real-valued functions $\phi_{i}, i=1,2, \ldots, m$, so that $T_{j}\left(\phi_{i} u\right)$ $-\phi_{i} T_{j} u=-\delta_{i j} u$ if $u$ satisfies the boundary conditions of (3.1). Define the functions $B_{j}$ of Theorem 1 by

$$
B_{j} u=\phi_{j} u \text { for } j=1,2, \ldots, m .
$$

The operators $B_{j}$ are symmetric in $L^{2}(\Omega)$ and leave the boundary conditions of (3.1) invariant. Moreover

$$
\left[T_{j}, B_{i}\right] u=-\delta_{i j} u .
$$

Proof. Using the formulation of $T_{j}$ in (3.6) we see that

$$
T_{j}(\phi u)-\phi T_{j} u=\mathbf{e}_{j} \cdot D U u \operatorname{grad} \phi
$$

for any differentiable function $\phi$. We thus need only show that there are functions $\phi_{i}$ so that

$$
\mathbf{e}_{j} \cdot D U \operatorname{grad} \phi_{i}=-\delta_{i j} .
$$

But (3.15) is equivalent to

$$
U^{-1} D \mathbf{e}_{j} \cdot \operatorname{grad} \phi_{i}=-\delta_{i j} .
$$

Thus the $\phi_{i}$ need only have constant gradient in a fixed direction since $U^{-1} D$ is a constant matrix. Such $\phi_{i}$ always exist. The remainder of the lemma follows easily.

We have enough now to prove

Theorem 2. If problem (3.1) has discrete spectrum $\lambda_{1} \leq \lambda_{2} \leq \cdots \leq \lambda_{n}<\lambda_{n+1}<$ .. and is selfadjoint with inner product (3.3) then the eigenvalues of (3.1) satisfy

$$
\sum_{i=1}^{n} \frac{\lambda_{i}}{\lambda_{n+1}-\lambda_{i}}>\frac{m n}{4}
$$

Proof. We take the $T_{j}$ and $B_{j}$ in Theorem 1 to be those of Lemmas 1 and 2. Evidently these $T_{j}$ and $B_{j}$ satisfy Proposition 1. With these $T_{j}$ and $B_{j},(2.1)$ becomes

$$
-n m \gamma+\gamma^{2} \sum_{i=1}^{n} \frac{\lambda_{i}}{\beta-\lambda_{i}}=-n m .
$$


This follows because

$$
\left\langle\left[T_{j}, B_{j}\right] u_{i}, u_{i}\right\rangle=-1, \quad \sum_{j=1}^{m}\left\langle T_{j} u_{i}, T_{j} u_{i}\right\rangle=\left\langle A u_{i}, u_{i}\right\rangle=\lambda_{i},
$$

and

$$
\frac{1}{2}\left\langle\left[\left[A, B_{j}\right], B_{j}\right] u_{i}, u_{i}\right\rangle=-1
$$

which follow from Lemma 2, Lemma 1, and Proposition 1 respectively. The left-hand side of (3.18) is a function of $\gamma$. In particular if we minimize the left-hand side of $(3.18)$ in $\gamma,(3.18)$ becomes

$$
-\frac{m^{2} n^{2}}{4 \sum_{i=1}^{n} \lambda_{i} /\left(\beta-\lambda_{i}\right)}=-m n
$$

the left-hand side of (3.19) being the minimum value of the left-hand side of (3.18) with

$$
\gamma=\frac{m n}{2 \sum_{i=1}^{n} \lambda_{i} /\left(\beta-\lambda_{i}\right)} .
$$

Rearrangement of (3.19) yields

$$
\sum_{i=1}^{n} \frac{\lambda_{i}}{\beta-\lambda_{i}}=\frac{m n}{4}
$$

There is always a (unique) solution $\beta>\lambda_{n}$ for (3.20). Moreover such a $\beta>$ $\lambda_{n+1}$ by Theorem 1 and Lemma 1 . Thus $\beta-\lambda_{i}>\lambda_{n+1}-\lambda_{i}$ and so

$$
\sum_{i=1}^{n} \frac{\lambda_{i}}{\lambda_{n+1}-\lambda_{i}}>\frac{m n}{4}
$$

Remark. Equation (3.1) includes all real constant coefficient second-order elliptic problems with $u=0$ on $\partial \Omega$. Writing out (3.1) yields

$$
A u=-\operatorname{div}\left(M^{2} \operatorname{grad} u\right)+M^{2} \mathbf{w} \cdot \operatorname{grad} u-\frac{1}{4}\|M \mathbf{w}\|^{2} u=\lambda u .
$$

Every real constant coefficient second-order elliptic operator can be written as

$$
E u=-\operatorname{div}\left(M^{2} \operatorname{grad} u\right)+\mathbf{v} \cdot \operatorname{grad} u+c u=\lambda u
$$

for constants $\mathbf{v} \in \mathbb{R}^{m}$ and $c \in \mathbb{R}$. Since $M^{2}$ is invertible we can always obtain $\mathbf{w}$ from $\mathbf{v}$ by $\mathbf{w}=\left(M^{2}\right)^{-1} \mathbf{v}$. Moreover, part of the constant $c$ may be incorporated into the eigenvalue $\lambda$. Thus we can rewrite (3.23) as

$$
\begin{aligned}
\widetilde{E} u & =-\operatorname{div}\left(M^{2} \operatorname{grad} u\right)+\mathbf{v} \cdot \operatorname{grad} u-\frac{1}{4}\left\|M^{-1} \mathbf{v}\right\|^{2} u \\
& =\left(\lambda-c+\frac{1}{4}\left\|M^{-1} \mathbf{v}\right\|^{2}\right) u=\mu u .
\end{aligned}
$$

Now $\widetilde{E}$ is in the form of (3.22) and its eigenvalues differ from those of (3.23) by the constant $-c+\frac{1}{4}\left\|M^{-1} \mathbf{v}\right\|^{2}$. Thus, as an easy corollary to Theorem 2 , the eigenvalues of (3.22) satisfy

$$
\sum_{i=1}^{n} \frac{\lambda_{i}-c+\frac{1}{4}\left\|M^{-1} \mathbf{v}\right\|^{2}}{\lambda_{n+1}-\lambda_{i}}>\frac{m n}{4}
$$

The result of Hile and Protter [2] is also an easy corollary of Theorem 2. 
Corollary 1. If the eigenvalues of

$$
-\Delta u=\lambda u \quad \text { in } \Omega \subseteq \mathbb{R}^{m} ; \quad u=0 \quad \text { on } \partial \Omega
$$

are discrete $\lambda_{1} \leq \lambda_{2} \leq \cdots \leq \lambda_{n}<\lambda_{n+1} \leq \cdots$ then

$$
\sum_{i=1}^{n} \frac{\lambda_{i}}{\lambda_{n+1}-\lambda_{i}}>\frac{m n}{4}
$$

Inequality (3.27) was only given as the weak inequality

$$
\sum_{i=1}^{n} \frac{\lambda_{i}}{\lambda_{n+1}-\lambda_{i}} \geq \frac{m n}{4}
$$

in [2] and, thus, has been strengthened here. Inequality (3.27) easily follows from Theorem 2 taking $M$ to be the identity matrix and $\mathbf{w}=\mathbf{0}$.

It is also possible to take the vector $\mathbf{w}$ in Theorem 2 to be complex, but then inner product (3.3) must be changed and the form of (3.1) must be changed somewhat. The following shows what to do in an easy problem with first-order terms with purely imaginary coefficients.

Theorem 3. Let $\Omega \subseteq \mathbb{R}^{m}$ be bounded. Let $\mathbf{w} \in \mathbb{R}^{m}$ be a constant vector. Consider the eigenvalue problem

$$
A u=-\Delta u-2 i \mathbf{w} \cdot \operatorname{grad} u=\lambda u \quad \text { in } \Omega, \quad u=0 \quad \text { on } \partial \Omega .
$$

The operator $A$ is symmetric in the inner product

$$
\langle u, v\rangle=\int_{\Omega} u \bar{v}
$$

subject to the boundary conditions of (3.28). If the eigenvalues of (3.28) are discrete, $\lambda_{1} \leq \lambda_{2} \leq \cdots \leq \lambda_{n}<\lambda_{n+1} \leq \cdots$, then the following inequality is satisfied:

$$
\sum_{i=1}^{n} \frac{\lambda_{i}-\|\mathbf{w}\|^{2}}{\lambda_{n+1}-\lambda_{i}}>\frac{m n}{4}
$$

Proof. The symmetry of $A$ is easy. Again we use Theorem 1 with

$$
T_{j} u=\frac{\partial u}{\partial x_{j}}+i w_{j} u
$$

and

$$
B_{j} u=-x_{j} u \text { for } j=1,2, \ldots, m,
$$

where $\mathbf{x}=\left(x_{1}, x_{2}, \ldots, x_{m}\right)$ and $\mathbf{w}=\left(w_{1}, w_{2}, \ldots, w_{m}\right)$. It is easy to see that the $T_{j}$ are all skew-symmetric and the $B_{j}$ are symmetric and preserve the boundary conditions. As before, we have $\left[T_{j}, B_{i}\right] u=-\delta_{i j} u$ and

$$
\begin{aligned}
\sum_{j=1}^{m}\left\langle T_{j} u, T_{j} u\right\rangle & =\sum_{j=1}^{m} \int_{\Omega}\left(\frac{\partial u}{\partial x_{j}}+i w_{j} u\right) \overline{\left(\frac{\partial u}{\partial x_{j}}+i w_{j} u\right)} \\
& =\int_{\Omega}\left(-\Delta u-2 i \mathbf{w} \cdot \operatorname{grad} u+\|\mathbf{w}\|^{2} u\right) \bar{u} \\
& =\langle A u, u\rangle+\|\mathbf{w}\|^{2}\langle u, u\rangle .
\end{aligned}
$$


With the foregoing and Proposition 1, equation (2.1) becomes (taking $\gamma$ as before)

$$
\sum_{i=1}^{n} \frac{\lambda_{i}+\|\mathbf{w}\|^{2}}{\lambda_{n+1}-\lambda_{i}}>\sum_{i=1}^{n} \frac{\lambda_{i}-\|\mathbf{w}\|^{2}}{\beta-\lambda_{i}}=\frac{m n}{4} .
$$

If an elliptic operator has any variable coefficients, a completely general solution is not easy. Several types of nonconstant coefficient problems are considered in the remainder of this section.

Theorem 4. Let $\Omega \subseteq \mathbb{R}^{m}$ be bounded and consider the eigenvalue problem

$$
A u=-\Delta u+\rho(x) u=\lambda u \quad \text { in } \Omega ; \quad u=0 \quad \text { on } \partial \Omega
$$

where $\rho$ is a nonnegative real-valued function on $\Omega$. If $\Omega$ is such that (3.34) is selfadjoint in inner product (3.29) and its eigenvalues, $\lambda_{1} \leq \lambda_{2} \leq \cdots \leq \lambda_{n}<$ $\lambda_{n+1} \leq \cdots$, are discrete then the inequality

$$
\sum_{i=1}^{n} \frac{\lambda_{i}}{\lambda_{n+1}-\lambda_{i}} \geq \frac{n m}{4}
$$

holds.

Proof. The operator $A$ is symmetric in the inner product of (3.29). For this problem we may choose

$$
\begin{gathered}
T_{j} u=\partial u / \partial x_{j} \quad \text { for } j=1,2, \ldots, m, \\
T_{m+1} u=(i \sqrt{\rho(x)}) u .
\end{gathered}
$$

Also we take

$$
\begin{gathered}
B_{j} u=-x_{j} u \text { for } j=1,2, \ldots, m, \\
B_{m+1} u=u .
\end{gathered}
$$

It is easily seen that the $T_{j}$ are skew-symmetric and the $B_{j}$ are symmetric with the given boundary conditions. We also have

$$
\sum_{j=1}^{m+1}\left\langle T_{j} u, T_{j} u\right\rangle=\langle A u, u\rangle .
$$

The commutators $\left[T_{j}, B_{j}\right] u=-u$ for $j=1,2, \ldots, m$ and $\left[T_{m+1}, B_{m+1}\right]=$ $0,\left[A, B_{j}\right]=2 T_{j}$ for $j=1,2, \ldots, m$ and $\left[A, B_{m+1}\right]=0$. With the above commutators, $(2.1)$ becomes

$$
\gamma(-n m)+\gamma^{2} \sum_{i=1}^{n} \frac{\lambda_{i}}{\beta-\lambda_{i}}=-n m .
$$

Since (3.39) matches (3.18) the remainder of the proof follows that of Theorem 2.

Theorem 4 may be extended to arbitrary functions $\rho$ as follows: 
Corollary 2. Suppose that the hypotheses of Theorem 4 hold except that we only have $\rho(x) \geq M$ for all $x \in \Omega$. (The constant $M$ may be either positive or negative.) Then the eigenvalues of (3.34) satisfy

$$
\sum_{i=1}^{n} \frac{\lambda_{i}-M}{\lambda_{n+1}-\lambda_{i}} \geq \frac{m n}{4}
$$

Proof. We have $\rho(x)-M \geq 0$ and thus

$$
\tilde{A} u=-\Delta u+(\rho(x)-M) u=(\lambda-M) u=\mu u
$$

is in the form of (3.34) and has eigenvalues $\mu_{i}=\lambda_{i}-M$. Since the eigenvalues $\mu_{i}$ satisfy (3.35), replacing $\mu_{i}$ with $\lambda_{i}-M$ yields the result (3.40).

Remark. Corollary 2 improves (3.35) even for $\rho(x) \geq 0$ provided that $\rho(x) \geq$ $M>0$ in $\Omega$.

In general it is difficult at best to establish results similar to the above for elliptic problems with variable coefficients which are differentiated. However in the case of an ordinary differential equation the solution is easy.

Theorem 5. Let $I=(\alpha, \beta) \subseteq \mathbb{R}$ be an open interval. Let $\rho(x)>0$ in $I$, and let $g(x)$ be any real-valued function on I. If the eigenvalues of the Sturm-Liouville problem

$$
A u=-\left((\rho(x))^{2} u^{\prime}\right)^{\prime}+g(x) u=\lambda u \quad \text { in } I, \quad u(\alpha)=u(\beta)=0
$$

are discrete $\lambda_{1} \leq \lambda_{2} \leq \cdots \leq \lambda_{n}<\lambda_{n+1} \leq \cdots$ and if $g-\frac{1}{4}\left(\left(\rho^{\prime}\right)^{2}-\left(\rho^{2}\right)^{\prime \prime}\right) \geq M$, a constant, on $I$ then the eigenvalues satisfy

$$
\sum_{i=1}^{n} \frac{\lambda_{i}-M}{\lambda_{n+1}-\lambda_{i}} \geq \frac{n}{4}
$$

Proof. The operator $A$ is symmetric in the inner product $\langle u, v\rangle=\int_{I} u \bar{v}$. Here we choose

$$
\begin{aligned}
& T_{1} u=\frac{1}{2}\left(\rho(x) u^{\prime}+(\rho(x) u)^{\prime}\right) \text { and } B_{1} u=\phi u, \\
& T_{2} u=\left(i \sqrt{g-\frac{1}{4}\left(\left(\rho^{\prime}\right)^{2}-\left(\rho^{2}\right)^{\prime \prime}\right)-M}\right) u \text { and } B_{2} u=u,
\end{aligned}
$$

where $\rho \phi^{\prime}=-1$. The operators $T_{j}$ are easily shown to be skew-symmetric and $B_{j}$ are clearly symmetric. We also have

$$
\begin{aligned}
\left\langle T_{1} u, T_{1} u\right\rangle & =\int_{I}\left|\rho u^{\prime}+\frac{1}{2} \rho^{\prime} u\right|^{2} \\
& =\int_{I}-\left(\rho^{2} u^{\prime}\right)^{\prime} \bar{u}+\frac{1}{4} \int_{I}\left(\left(\rho^{\prime}\right)^{2}-\left(\rho^{2}\right)^{\prime \prime}\right)|u|^{2}
\end{aligned}
$$

and

$$
\left\langle T_{2} u, T_{2} u\right\rangle=\int_{I}\left(g-\frac{1}{4}\left(\left(\rho^{\prime}\right)^{2}-\left(\rho^{2}\right)^{\prime \prime}\right)-M\right)|u|^{2} .
$$


Thus by equations $(3.45)$ we see that

$$
\left\langle T_{1} u, T_{1} u\right\rangle+\left\langle T_{2} u, T_{2} u\right\rangle=\langle A u, u\rangle-M\langle u, u\rangle .
$$

It is easy to see that

$$
\begin{gathered}
{\left[T_{1}, B_{1}\right] u=-u,} \\
{\left[T_{2}, B_{2}\right] u=0,} \\
{\left[A, B_{1}\right] u=2 T_{1} u,} \\
{\left[A, B_{2}\right] u=0 .}
\end{gathered}
$$

Combining equations (3.47) and putting them into (2.1) we get

$$
-\gamma n+\gamma^{2} \sum_{i=1}^{n} \frac{\lambda_{i}-M}{\lambda_{n+1}-\lambda_{i}}=-n \text {. }
$$

This is in the same form as (3.18) with $m=1$. Again the remainder follows the proof of Theorem 2 .

The result of Theorem 5 can be partially extended to certain variable coefficient elliptic problems but not to the problem of Theorem 2 if the matrix $M$ has variable coefficients. In the general case there is no guarantee that the required system of first-order partial differential equations can be solved. Of course these solutions have been used to construct the operators $B_{j}$. However we are able to extend the idea to certain diagonal elliptic problems.

Theorem 6. Let $\Omega \subseteq \mathbb{R}^{m}$ be a bounded region in which the problem

$$
A u=-\sum_{j=1}^{m} \frac{\partial}{\partial x_{j}}\left(d_{j}^{2} \frac{\partial u}{\partial x_{j}}\right)+g u=\lambda u \quad \text { in } \Omega ; \quad u=0 \quad \text { on } \partial \Omega
$$

has discrete spectrum $\lambda_{1} \leq \lambda_{2} \leq \cdots \leq \lambda_{n}<\lambda_{n+1} \leq \cdots$. In (3.49) the coefficient functions $d_{j}$ are assumed to be positive and differentiable functions of $x_{j}$ only and $g$ is any function on $\Omega$. If $M$ is a real constant so that

$$
g(x)-\frac{1}{4} \sum_{j=1}^{m}\left(\left(\frac{\partial d_{j}}{\partial x_{j}}\right)^{2}-\frac{\partial^{2}}{\partial x_{j}^{2}}\left(d_{j}^{2}\right)\right) \geq M
$$

in $\Omega$, then the eigenvalues satisfy

$$
\sum_{i=1}^{n} \frac{\lambda_{i}-M}{\lambda_{n+1}-\lambda_{i}} \geq \frac{m n}{4}
$$

Proof. In Theorem 1 we take

$$
T_{j} u=d_{j} \frac{\partial u}{\partial x_{j}}+\frac{1}{2} \frac{\partial d_{j}}{\partial x_{j}} u \text { for } j=1,2, \ldots, m
$$


and

$$
T_{m+1} u=\left(i \sqrt{g-\frac{1}{4} \sum_{j=1}^{m}\left(\left(\frac{\partial d_{j}}{\partial x_{j}}\right)^{2}-\frac{\partial^{2}}{\partial x_{j}^{2}}\left(d_{j}^{2}\right)\right)-M}\right) u .
$$

It is easy to show that the operator $A$ is symmetric in the inner product of (3.29) and the operators of (3.51) are skew-symmetric. We choose the operators $B_{j}$ by

$$
B_{j} u=\phi_{j} u \text { for } j=1,2, \ldots, m,
$$

where $d_{j} \partial \phi_{j} / \partial x_{j}=-1$ in $\Omega, \partial \phi_{j} / \partial x_{k}=0$ if $k \neq j$ and

$$
B_{m+1} u=-u \text {. }
$$

Such $\phi_{j}$ exist since the $d_{j}$ depend only on $x_{j}$. The operators $B_{j}$ of (3.52) are seen to be symmetric in the inner product (3.29). We also have

$$
\left\langle T_{j} u, T_{j} u\right\rangle=-\int_{\Omega} \frac{\partial}{\partial x_{j}} d_{j}^{2} \frac{\partial u}{\partial x_{j}} \bar{u}-\frac{1}{4} \int_{\Omega}\left(\frac{\partial^{2}\left(d_{j}^{2}\right)}{\partial x_{j}^{2}}-\left(\frac{\partial d_{j}}{\partial x_{j}}\right)^{2}\right)|u|^{2}
$$

for $j=1,2, \ldots, m$ and

$$
\left\langle T_{m+1} u, T_{m+1} u\right\rangle=\int_{\Omega}\left(g-\frac{1}{4} \sum_{j=1}^{m}\left(\left(\frac{\partial d_{j}}{\partial x_{j}}\right)^{2}-\frac{\partial^{2}}{\partial x_{j}^{2}}\left(d_{j}\right)^{2}\right)-M\right)|u|^{2} .
$$

Hence

$$
\sum_{j=1}^{m+1}\left\langle T_{j} u, T_{j} u\right\rangle=\langle A u, u\rangle-M\langle u, u\rangle .
$$

Looking at the $B_{j}$ we have

$$
\left\langle\left[T_{j}, B_{j}\right] u, u\right\rangle=-\langle u, u\rangle \text { for } j=1,2, \ldots, m
$$

and

$$
\left\langle\left[T_{m+1}, B_{m+1}\right] u, u\right\rangle=0 .
$$

Finally we have

$$
\sum_{j=1}^{m+1}\left\langle\left[\left[A, B_{j}\right] B_{j}\right] u, u\right\rangle=-2 m\langle u, u\rangle .
$$

With the results of (3.53)-(3.55), equation (2.1) becomes

$$
\gamma(-n m)+\gamma^{2} \sum_{i=1}^{n} \frac{\lambda_{i}-M}{\beta-\lambda_{i}}=-m n .
$$

Equation (3.56) is in the same form as (3.18) and the result follows as before. 
It is not clear whether a similar result may be obtained directly from (2.1) if any of the functions $d_{j}$ are allowed to depend on any coordinate other than $x_{j}$.

The results of this section are all implicit bounds on the eigenvalues of the various problems. Each give rise to explicit bounds. Inequalities (3.17) and (3.35) each imply that

$$
\lambda_{n+1} \leq \lambda_{n}+\frac{4}{m n} \sum_{i=1}^{n} \lambda_{i}
$$

since the sum in each is bounded above by

$$
\sum_{i=1}^{n} \frac{\lambda_{i}}{\lambda_{n+1}-\lambda_{n}} \geq \sum_{i=1}^{n} \frac{\lambda_{i}}{\lambda_{n+1}-\lambda_{i}} \geq \frac{m n}{4} .
$$

Factoring the constant $1 /\left(\lambda_{n+1}-\lambda_{n}\right)$ out of the sum in (3.58) and rearranging yields the desired result. Similarly inequality (3.30) implies that

$$
\lambda_{n+1}<\lambda_{n}+\frac{4}{m n} \sum_{i=1}^{n} \lambda_{i}-\frac{4\|\mathbf{w}\|^{2}}{m} .
$$

The other results of this section yield similar types of explicit inequalities.

Notice that each problem considered in this section has the same boundary condition: $u=0$ on $\partial \Omega$. This was necessary since the $B_{j}$ always turned out to be multiplication operators and this boundary condition is the only one preserved under multiplication by arbitrary functions. The fact that the $T_{j}$ are first-order differential operators for the most part and the $B_{j}$ multiplication operators seems to be basic and this author has not yet found any situation where Theorem 1 provides a useful application where this is not the case. In the following section we consider some higher-order problems.

\section{HIGHER-ORDER PROBLEMS}

So far we have only seen applications in which the sum $\sum_{j=1}^{M}\left\langle T_{j} u, T_{j} u\right\rangle=$ $\langle A u, u\rangle+\operatorname{constant}\langle u, u\rangle$. For higher-order problems this does not turn out to be the case. The results in this section depend on a theorem of this author [5] as follows.

Proposition 2. Let $V$ be a real or complex inner product space with inner product $(\cdot, \cdot)$. Let $D$ be a linear submanifold of $V$ and let $Q: D \rightarrow V$ be a linear operator in $V$. Let $n$ be a positive integer and let $u$ be a fixed vector in the domain of $Q^{n}$. Suppose that for all integers $r$ and $p$ which satisfy $0 \leq r \leq p \leq$ $n$, the equality $\left|\left(Q^{p} u, u\right)\right|=\left|\left(Q^{p-r} u, Q^{r} u\right)\right|$ holds. Then (i) for each integer $k$ and even integer $m$ which satisfy $0 \leq k \leq m \leq n$, the inequality

$$
\left|\left(Q^{k} u, u\right)\right| \leq\left|\left(Q^{m} u, u\right)\right|^{k / m}(u, u)^{(m-k) / m}
$$

holds. 
(ii) Inequality (4.1) is satisfied for each integer $k$ and odd integer $m$ which satisfy $0 \leq k \leq m \leq n$ if in addition to the above hypotheses there is a finite collection of operators $\left\{T_{j}: j=1,2, \ldots, M\right\}$ in $V$ which satisfy the following: For each pair of integers $r$ and $p$ so that $1 \leq r \leq p \leq n$ the equality

$$
\left|\left(Q^{p} u, u\right)\right|=\left|\sum_{j=1}^{M}\left(T_{j} Q^{p-r} u, T_{j} Q^{r-1} u\right)\right|
$$

holds.

Proof. See [5] or [4].

The result of Proposition 2 can be used in conjunction wth Theorem 1 after observing the following.

Proposition 3. Suppose that $M$ is a positive integer and that $Q, T_{j}$, and $B_{j}$, $j=1,2, \ldots, M$, are linear operators in an inner product space. If the commutators $\left[Q, T_{j}\right]=0$ and $\left[T_{j}, B_{i}\right]=-\delta_{i j}$ for $i, j=1,2, \ldots, M$ and if $Q=-\sum_{j=1}^{M} T_{j}^{2}$ then for all positive integers $n$,

$$
\sum_{j=1}^{M}\left[\left[Q^{n}, B_{j}\right], B_{j}\right]=-2 n(M+2(n-1)) Q^{n-1} .
$$

Proof. First we claim that

$$
\left[Q^{n}, B_{j}\right]=2 n Q^{n-1} T_{j}
$$

for all $j=1,2, \ldots, M$ and all positive $n$. We proceed by induction on $n$ for a fixed $j$. We see that $\left[Q^{1} B_{j}\right]=2 Q^{0} T_{j}=2 T_{j}$ in the proof of Proposition 1. For the inductive step notice that if (4.3) holds for some $n$ then

$$
\begin{aligned}
{\left[Q^{n+1}, B_{j}\right] } & =Q\left[Q^{n}, B_{j}\right]+\left[Q, B_{j}\right] Q^{n} \\
& =2 Q n Q^{n-1} T_{j}+2 T_{j} Q^{n}=2(n+1) Q^{n} T_{j}
\end{aligned}
$$

as required. By (4.3) we have

$$
\begin{aligned}
\sum_{j=1}^{M}\left[\left[Q^{n}, B_{j}\right], B_{j}\right]=\sum_{j=1}^{M}\left[2 n Q^{n-1} T_{j}, B_{j}\right] \\
\quad=2 n Q^{n-1} \sum_{j=1}^{M}\left[T_{j}, B_{j}\right]+2 n \sum_{j=1}^{M}\left[Q^{n-1}, B_{j}\right] T_{j} \\
\quad=-2 n M Q^{n-1}+2 n \sum_{j=1}^{M}\left(2(n-1) Q^{n-2} T_{j} T_{j}\right) \\
\quad=-2 n M Q^{n-1}-4 n(n-1) Q^{n-1}=-2 n(M+2(n-1)) Q^{n-1}
\end{aligned}
$$

Proposition 3 allows us to take the operator $A$ in Theorem 1 to be $Q^{n}$ and if we can write $Q=-\sum_{j=1}^{M} T_{j}^{2}$ where the $T_{j}$ are skew-symmetric, we can 
use Proposition 2 to obtain a suitable estimate for the right-hand side of (2.1). This enables us to get results similar to those of $\S 3$ for higher-order problems. Our principal application is to polynomials in the Laplacian. We restrict our consideration to the Laplacian for simplicity even though the Laplacian can be replaced with the operator of (3.1). Several earlier results by Hile and Yeh [3] and Chen [1] were established for certain even degree monomials in the Laplacian of the form

$$
\Delta^{2 l} u=\lambda u \quad \text { in } \Omega \subseteq \mathbb{R}^{m}, \quad u=\frac{\partial u}{\partial n}=\cdots=\frac{\partial^{2 l-1} u}{\partial n^{2 l-1}}=0 \quad \text { on } \partial \Omega,
$$

where $l$ is one of a class of positive integers. This author [4] later improved and extended the earlier results of problem (4.6) to arbitrary polynomials in the Laplacian using Proposition 2.

Theorem 7. Let $p(x)=\sum_{i=0}^{l} a_{i} x^{i}$ be a monic polynomial and $q(x)=\sum_{i=0}^{r} b_{i} x^{i}$ be a polynomial. Suppose that $a_{i} \geq 0$ and $b_{i} \geq 0$ for all $i$ and $r<l$. Suppose that $\Omega \subseteq \mathbb{R}^{m}$ is a region in which the problem

$$
\begin{gathered}
(p(-\Delta)-q(-\Delta)) u=\lambda u \quad \text { in } \Omega, \\
u=\frac{\partial u}{\partial n}=\cdots=\frac{\partial^{l-1} u}{\partial n^{l-1}}=0 \quad \text { on } \partial \Omega
\end{gathered}
$$

is selfadjoint with discrete spectrum $\lambda_{1} \leq \lambda_{2} \leq \cdots \leq \lambda_{n}<\lambda_{n+1} \leq \cdots$. Then the eigenvalues of (4.7) satisfy

$$
\left(\sum_{i=1}^{n} \frac{\alpha_{i, 1}}{\lambda_{n+1}-\lambda_{i}}\right)\left(\sum_{i=1}^{n} \sum_{k=1}^{l}(m+2 k-2) k a_{k} \alpha_{i, k-1}^{k-1}\right)>\frac{m^{2} n^{2}}{4} .
$$

In (4.8) we define $\alpha_{i, 0}=1$ and for $j \geq 1, \alpha_{i, j}$ is the largest (positive) root of

$$
a_{0}+\sum_{k=j}^{l} a_{k} x^{k}-q(x)-\lambda_{i}=0 \quad \text { if } r \leq j \leq l,
$$

$$
a_{0}+\sum_{k=j+1}^{r} b_{k} \alpha_{i, k}^{k}-\sum_{k=j}^{l} a_{k} x^{k}-\sum_{k=0}^{j} b_{k} x^{k}-\lambda_{i}=0 \quad \text { if } 1 \leq j \leq r-1 .
$$

Proof. Here we choose the operator $Q$ of Propositions 2 and 3 by $Q=-\Delta$, $T_{j} u=\partial u / \partial x_{j}$ and $B_{j} u=-x_{j} u$. Finally we choose the operator $A$ of Theorem 1 to be $A=p(-\Delta)-q(-\Delta)=p(Q)-q(Q)$. These operators satisfy all of the hypotheses of Theorem 1 and Propositions 2 and 3 so (2.1) becomes

$$
-\gamma n m+\gamma^{2} \sum_{i=1}^{n} \frac{\left\langle Q u_{i}, u_{i}\right\rangle}{\beta-\lambda_{i}}=-\frac{1}{2} \sum_{i=1}^{n}\left\langle\left(2 m(p-q)^{\prime}(Q)+4 Q(p-q)^{\prime \prime}(Q)\right) u_{i}, u_{i}\right\rangle .
$$

First we want to find a lower bound for the right-hand side of (4.10). Proposition 2 shows that $\left\langle Q^{k} u_{i}, u_{i}\right\rangle \leq\left\langle Q^{j} u_{i}, u_{i}\right\rangle^{k / j}$ if $0 \leq k \leq j \leq l$. Thus if we fix 
$i$ and let $\beta_{k}^{k}=\left\langle Q^{k} u_{i}, u_{i}\right\rangle$ for $k=1,2, \ldots, l$, Proposition 2 implies that $0 \leq \beta_{1} \leq \beta_{2} \leq \cdots \leq \beta_{l}$, where $\beta_{j}$ is the principal root of $\beta_{j}^{j}$. (We take $\beta_{0}=1=\left\langle Q^{0} u_{i}, u_{i}\right\rangle$.)

For $j \geq r$ we have

$$
\begin{aligned}
\lambda_{i} & =\sum_{k=0}^{l} a_{k} \beta_{k}^{k}-\sum_{k=0}^{r} b_{k} \beta_{k}^{k} \geq \sum_{k=j}^{l} a_{k} \beta_{k}^{k}+a_{0}-\sum_{k=0}^{r} b_{k} \beta_{k}^{k} \\
& \geq \sum_{k=j}^{l} a_{k} \beta_{j}^{k}+a_{0}-\sum_{k=0}^{r} b_{k} \beta_{j}^{k}=\sum_{k=j}^{l} a_{k} \beta_{j}^{k}+a_{0}-q\left(\beta_{j}\right) .
\end{aligned}
$$

Now consider the functions $f_{j}(x)=a_{0}+\sum_{k=j}^{l} a_{k} x^{k}-q(x)$ for $r \leq j \leq l$. Now $f_{j}(x) \rightarrow+\infty$ as $x \rightarrow+\infty$ and is continuous and (4.11) asserts that $f_{j}\left(\beta_{j}\right) \leq \lambda_{i}$. The continuity of $f$ and limiting behavior imply the existence of a number which we have called $\alpha_{i, j}$ so that $f_{j}\left(\alpha_{i, j}\right)=\lambda_{i}$ and for this root we have $\alpha_{i, j} \geq \beta_{j}$.

For $1 \leq j \leq r-1$ we have

$$
\begin{aligned}
\lambda_{i} & =\sum_{k=0}^{l} a_{k} \beta_{k}^{k}-\sum_{k=0}^{r} b_{k} \beta_{k}^{k} \\
& =\sum_{k=0}^{l} a_{k} \beta_{k}^{k}-\sum_{k=0}^{j} b_{k} \beta_{k}^{k}-\sum_{k=j+1}^{r} b_{k} \beta_{k}^{k} \\
& \geq \sum_{k=j}^{l} a_{k} \beta_{j}^{k}+a_{0}-\sum_{k=0}^{j} b_{k} \beta_{j}^{k}-\sum_{k=j+1}^{r} b_{k} \alpha_{i, k}^{k},
\end{aligned}
$$

where the $\alpha_{i, k}$ are those found from (4.9b). The argument that (4.12) leads to $(4.9 \mathrm{~b})$ follows the argument for $(4.9 \mathrm{a})$ above.

Now

$$
\begin{aligned}
& \left\langle\left(m(p-q)^{\prime}(Q)+2 Q(p-q)^{\prime \prime}(Q)\right) u_{i}, u_{i}\right\rangle \leq\left\langle\left(m p^{\prime}(Q)+2 Q p^{\prime \prime}(Q)\right) u_{i}, u_{i}\right\rangle \\
& \quad=m \sum_{k=1}^{l} k a_{k} \beta_{k-1}^{k-1}+2 \sum_{k=1}^{l} k(k-1) a_{k} \beta_{k-1}^{k-1} \\
& \quad \leq \sum_{k=1}^{l} k(m+2 k-2) a_{k} \alpha_{i, k-1}^{k-1} .
\end{aligned}
$$

Inequality (4.13) yields the desired lower bound for the right-hand side of $(4.10)$ and $\alpha_{i, 1} \geq\left\langle Q u_{i}, u_{i}\right\rangle$. Thus (4.10) can be rewritten

$$
-\gamma m n+\gamma^{2} \sum_{i=1}^{n} \frac{\alpha_{i, 1}}{\beta-\lambda_{i}} \geq-\sum_{i=1}^{n} \sum_{k=1}^{l} k(m+2 k-2) a_{k} \alpha_{i, k-1}^{k-1} .
$$

In (4.14), $\beta$ is the same number which yields equality in (4.10). Evidently equality is attained in (4.14) for a larger value of $\beta$. In particular if $\gamma>0$ and 
$\beta>\lambda_{n}$ yield equality in (4.14) we must have $\beta \geq \lambda_{n+1}$. Again, minimizing the left-hand side of (4.14) in $\gamma$ as in Proposition 2 and rearranging yields the desired result (4.8). As before, the strict inequality comes from the fact that for each $j, \partial u_{i} / \partial x_{j}$ will not satisfy the boundary conditions of $(4.7 \mathrm{~b})$ if $u_{i}$ is an eigenvector of $(4.7 \mathrm{a})$.

The result (4.8) is at best difficult to understand along with the computation of the $\alpha_{i, k}$. A more explicit form of (4.8) is available if we take $q=0$.

Theorem 8. Suppose the hypotheses of Theorem 7 except that $q=0$ and $a_{0}=$ 0 . Then the eigenvalues satisfy

$$
\left(\sum_{i=1}^{n} \frac{\lambda_{i}^{1 / l}}{\lambda_{n+1}-\lambda_{i}}\right)\left(\sum_{i=1}^{n} \frac{m}{2} p^{\prime}\left(\lambda_{i}^{1 / l}\right)+\lambda_{i}^{1 / l} p^{\prime \prime}\left(\lambda_{i}^{1 / l}\right)\right)>\frac{m^{2} n^{2}}{8} .
$$

Proof. In the notation of the proof of Theorem 7 we see that when $j=l$, (4.11) reduces to

$$
\lambda_{i} \geq \beta_{l}^{l} .
$$

Thus $\beta_{l} \leq \lambda_{i}^{1 / l}$. Also for $0 \leq j \leq l$ we have $\beta_{j}^{j} \leq \beta_{l}^{j}$. (The extension to $j=0$ is easy since $1 \leq 1$.) Finally $\beta_{l} \leq \alpha_{i, l} \leq \lambda_{i}^{1 / l}$. Writing out the right-hand side of (4.14) with $\alpha_{i, j}=\alpha_{i, l}$ for all $j, 0 \leq j \leq l$, expressing it in terms of derivatives of $p$ and following the remainder of the proof of Theorem 7 yields the result.

At this point we look at a corollary of Theorem 8 and show that the earlier results of Hile and Yeh [3] and Chen [1] follow from this corollary.

Corollary 3. In Theorem 8 if $p(x)=x^{l}, l>0$ then the inequality

$$
\left(\sum_{i=1}^{n} \frac{\lambda_{i}^{1 / l}}{\lambda_{n+1}-\lambda_{i}}\right)\left(\sum_{i=1}^{n} \lambda_{i}^{(l-1) / l}\right)>\frac{m^{2} n^{2}}{4 l(m+2 l-2)}
$$

is satisfied.

Proof. Comparison of (4.17) and (4.15) shows that we need only show that for $p(x)=x^{l}$ we have

$$
\frac{m}{2} p^{\prime}\left(\lambda_{i}^{1 / l}\right)+\lambda_{i}^{1 / l} p^{\prime \prime}\left(\lambda_{i}^{1 / l}\right)=\frac{1}{2} \lambda_{i}^{(l-1) / l} l(m+2 l-2) .
$$

This is an easy task.

The results of [3] and [1] are as follows.

Proposition 4. For certain even integers $l$ (the condition is irrelevant here) the eigenvalues of the problem in Corollary 3 satisfy

$$
\left(\sum_{i=1}^{n} \frac{\lambda_{i}^{1 / l}}{\lambda_{n+1}-\lambda_{i}}\right)\left(\sum_{i=1}^{n} \lambda_{i}\right)^{(l-1) / l} n^{1 / l} \geq \frac{m^{2} n^{2}}{4 l(m+2 l-2)} .
$$


The result (4.19) follows easily from (4.17) by way of the Hölder inequality which for our purposes reads:

$$
\sum_{i=1}^{n} \lambda_{i}^{(l-1) / l} \leq n^{1 / l}\left(\sum_{i=1}^{n} \lambda_{i}\right)^{(l-1) / l} .
$$

The theorems and corollaries above also hold if the Laplacian, at least $-\Delta$, is replaced with the operator of Theorem 2. Furthermore, similar results can be obtained for polynomials in the operator of Theorem 3. The problem of Theorem 4 presents an interesting possibility.

Theorem 9. Let $p(x)$ be a monic polynomial of degree $l>0$ with nonnegative coefficients and zero constant term, $\Omega \subseteq \mathbb{R}^{m}$ and $\rho$ any function and $M a$ constant with $\rho \geq M$ on $\Omega$. If $\Omega$ is such that the problem

$$
\text { (4.21) } p(-\Delta) u+\rho(x) u=\lambda u \quad \text { in } \Omega ; \quad u=\frac{\partial u}{\partial n}=\cdots=\frac{\partial^{l-1} u}{\partial n^{l-1}}=0 \quad \text { on } \partial \Omega
$$

is selfadjoint with discrete spectrum $\lambda_{1} \leq \lambda_{2} \leq \cdots \leq \lambda_{n}<\lambda_{n+1} \leq \cdots$ then the eigenvalues of (4.21) satisfy

$$
\begin{aligned}
& \left(\sum_{i=1}^{n} \frac{\left(\lambda_{i}-M\right)^{1 / l}}{\lambda_{n+1}-\lambda_{i}}\right)\left(\sum_{i=1}^{n} m p^{\prime}\left(\left(\lambda_{i}-M\right)^{1 / l}\right)+2\left(\lambda_{i}-M\right)^{1 / l} p^{\prime \prime}\left(\left(\lambda_{i}-M\right)^{1 / l}\right)\right) \\
& \quad>\frac{m^{2} n^{2}}{4}
\end{aligned}
$$

if $\lambda_{1}-M \geq 0$.

Proof. Here in Propositions 2 and 3 we take $T_{j}$ and $B_{j}$ as in Theorem 4 except that $B_{m+1}=T_{m+1}=0, Q=-\Delta$ and in Theorem $1, A u=(p(-\Delta)+\rho(x)) u$. Proposition 3 is not directly applicable in this case. However as before we have

$$
\left[\left[A, B_{j}\right], B_{j}\right]=\left[\left[p(-\Delta), B_{j}\right], B_{j}\right] \text { for } j=1,2, \ldots, m+1 .
$$

Since the $B_{j}$ and $Q=-\Delta$ satisfy the hypotheses of Proposition 3 we get

$$
\begin{gathered}
\sum_{j=1}^{m}\left\langle\left[\left[A, B_{j}\right], B_{j}\right] u_{i}, u_{i}\right\rangle=\sum_{j=1}^{m}\left\langle\left[\left[p(-\Delta), B_{j}\right], B_{j}\right] u_{i}, u_{i}\right\rangle \\
=-2\left\langle\left(m p^{\prime}(-\Delta)+2(-\Delta) p^{\prime \prime}(-\Delta)\right) u_{i}, u_{i}\right\rangle .
\end{gathered}
$$

Also

$$
\sum_{j=1}^{m}\left\langle\left[T_{j}, B_{j}\right] u_{i}, u_{i}\right\rangle=-m\left\langle u_{i}, u_{i}\right\rangle
$$

and

$$
\sum_{j=1}^{m}\left\langle T_{j} u_{i}, T_{j} u_{i}\right\rangle=\left\langle(-\Delta) u_{i}, u_{i}\right\rangle .
$$


Putting the results of (4.24)-(4.26) into (2.1) leaves us with

$$
\gamma(-n m)+\gamma^{2} \sum_{i=1}^{n} \frac{\left\langle(-\Delta) u_{i}, u_{i}\right\rangle}{\beta-\lambda_{i}}=-\left\langle\left(m p^{\prime}(-\Delta)+2(-\Delta) p^{\prime \prime}(-\Delta)\right) u_{i}, u_{i}\right\rangle
$$

By the proof of Theorem 7 for each $i$ we have

$$
\begin{aligned}
\left\langle(-\Delta)^{k} u_{i}, u_{i}\right\rangle^{l / k} & \leq\left\langle(-\Delta)^{l} u_{i}, u_{i}\right\rangle \leq\left\langle p(-\Delta) u_{i}, u_{i}\right\rangle \\
& =\left\langle\left(\lambda_{i}-\rho\right) u_{i}, u_{i}\right\rangle \leq\left\langle\left(\lambda_{i}-M\right) u_{i}, u_{i}\right\rangle=\lambda_{i}-M
\end{aligned}
$$

for $1 \leq k \leq l$. Thus

$$
\left\langle-\Delta u_{i}, u_{i}\right\rangle \leq\left(\lambda_{i}-M\right)^{1 / l}
$$

and

$$
\begin{aligned}
& \left\langle\left(m p^{\prime}(-\Delta)+2(-\Delta) p^{\prime \prime}(-\Delta)\right) u_{i}, u_{i}\right\rangle \\
& \quad \leq m p^{\prime}\left(\left(\lambda_{i}-M\right)^{1 / l}\right)+2\left(\lambda_{i}-M\right)^{1 / l} p^{\prime \prime}\left(\left(\lambda_{i}-M\right)^{1 / l}\right) .
\end{aligned}
$$

Placing the estimates of (4.29) into (4.27), using the same argument as was used in the proof of Theorem 7 yields the result.

Remarks. (i) In Theorem 9 if $M=\inf \{\rho(x): x \in \Omega\}$, the quantities $\lambda_{1}-M \geq 0$ in $\Omega$ is always satisfied.

(ii) A sharper estimate can be attained in (4.28) as is done in the proof of Theorem 7, but we have chosen the simpler argument for the sake of simplicity.

The operators of Theorems 2 and 3 can be combined into higher-order problems through a polynomial as above as well as the operators of Theorems 5 and 6 but the number of combinations is so large that it is hopeless to list all the possibilities as well as the variety of estimates available. However a problem for which Theorem 1 is not directly applicable but which follows as a corollary to our earlier problems is

Corollary 4. Let $p(x)$ be a polynomial with nonnegative coefficients of degree $l>0$ and $\Omega \subseteq \mathbb{R}^{m}$ a bounded domain. If $\Omega$ is such that

$$
\begin{gathered}
p(-\Delta) u=\lambda u \quad \text { in } \Omega ; \\
u=\Delta u=\Delta^{2} u=\cdots=\Delta^{1-1} u=0 \quad \text { on } \partial \Omega
\end{gathered}
$$

is selfadjoint with discrete spectrum then

$$
\sum_{i=1}^{n} \frac{\alpha_{i}}{\alpha_{n+1}-\alpha_{i}}>\frac{m n}{4}
$$

where $\alpha_{i}$ is the (only) positive number so that $p\left(\alpha_{i}\right)=\lambda_{i}$.

Proof. The eigenvalues, $\lambda_{i}$ of (4.30), (4.31) satisfy

$$
p\left(\alpha_{i}\right)=\lambda_{i},
$$

where $\alpha_{i}$ is the $i$ th eigenvalue of the problem of (3.26). The result (4.32) then follows easily from Corollary 1. 
A variety of other boundary conditions for problem (4.30) simply yield problems whose eigenvalues are some function of the eigenvalues of one of the earlier problems in either of $\S \S 3$ or 4 . Each such problem yields a result similar to that of Corollary 4. As a final note for higher-order problems, explicit bounds can again be established from the implicit bounds given here. For example, (4.15) yields, by the same argument as was used to establish (3.57) and (3.59),

$$
\lambda_{n+1}<\lambda_{n}+\frac{8}{m^{2} n^{2}}\left(\sum_{i=1}^{n} \lambda_{i}^{1 / l}\right)\left(\sum_{i=1}^{n} \frac{m}{2} p^{\prime}\left(\lambda_{i}^{1 / l}\right)+\lambda_{i}^{1 / l} p^{\prime \prime}\left(\lambda_{i}^{1 / l}\right)\right) .
$$

So we see that for the type of higher-order problem considered here the rate of growth in the eigenvalues is bounded above in a way which has no explicit dependence on the domain $\Omega$.

\section{A SYSTEM OF EQUATIONS}

Our last task is to show how to apply Theorem 1 to a system. Let $\alpha$ be a constant, $\alpha \geq 0$. We consider the eigenvalue problem

$$
A \mathbf{u}=-\Delta \mathbf{u}-\alpha \operatorname{grad}(\operatorname{div}(\mathbf{u}))=\Lambda \mathbf{u} \quad \text { in } \Omega, \quad \mathbf{u}=\mathbf{0} \quad \text { on } \partial \Omega
$$

which governs the behavior of an elastic medium. We begin with a few preliminaries.

Lemma 3. In the inner product $\langle\mathbf{u}, \mathbf{v}\rangle=\int_{\Omega} \mathbf{u} \cdot \mathbf{v}$, which we call $L^{2}(\Omega)$, define the operators

$$
R_{j} \mathbf{u}=\operatorname{div}(\mathbf{u}) \operatorname{grad} x_{j}+\operatorname{grad} u_{j} \quad \text { for } j=1,2, \ldots, m
$$

and

$$
S_{j} \mathbf{u}=\frac{\partial \mathbf{u}}{\partial x_{j}} \quad \text { for } j=1,2, \ldots, m,
$$

where $\mathbf{u}=\left(u_{1}, u_{2}, \ldots, u_{m}\right)$ and $\mathbf{u}=\mathbf{0}$ on $\partial \Omega$. The symbol $x_{j}$ is the projection of a vector $\mathbf{x}$ onto its $j$ th component. The operators $R_{j}$ and $S_{j}$ satisfy

$$
\sum_{j=1}^{m}\left\langle R_{j} \mathbf{u}, R_{j} \mathbf{u}\right\rangle=-\langle(m+2) \operatorname{grad} \operatorname{div} \mathbf{u}+\Delta \mathbf{u}, \mathbf{u}\rangle
$$

and

$$
\sum_{j=1}^{m}\left\langle S_{j} \mathbf{u}, S_{j} \mathbf{u}\right\rangle=-\langle\Delta \mathbf{u}, \mathbf{u}\rangle
$$

Proof. If $\mathbb{R}^{m}$ we have

$$
\operatorname{div}(g \mathbf{u})=\operatorname{grad} g \cdot \mathbf{u}+g \operatorname{div} \mathbf{u}
$$

and the divergence theorem

$$
\int_{\Omega} \operatorname{div} \mathbf{u}=\int_{\partial \Omega} \mathbf{u} \cdot d S
$$


If $\mathbf{u}=\mathbf{0}$ on $\partial \Omega$ then (5.5) and (5.6) may be combined to yield

$$
\int_{\Omega} \operatorname{grad} g \cdot \mathbf{u}=-\int_{\Omega} g \operatorname{div} \mathbf{u} .
$$

The definition of $R_{j}$ shows that

$$
\begin{aligned}
\left\langle R_{j} \mathbf{u}, R_{j} \mathbf{u}\right\rangle & =\int_{\Omega}\left\|\operatorname{div} \mathbf{u g r a d} x_{j}+\operatorname{grad} u_{j}\right\|^{2} \\
& =\int_{\Omega}(\operatorname{div} \mathbf{u})^{2}+2 \int_{\Omega} \operatorname{div} \mathbf{u} \frac{\partial u_{j}}{\partial x_{j}}+\int_{\Omega}\left\|\operatorname{grad} u_{j}\right\|^{2} .
\end{aligned}
$$

Application of (5.7) with the identification $\mathbf{u} \sim \operatorname{grad} u_{j}$ and $g \sim u_{j}$ we see that

$$
\int_{\Omega}\left\|\operatorname{grad} u_{j}\right\|^{2}=-\int_{\Omega}\left(\operatorname{div} \operatorname{grad} u_{j}\right) u_{j}=-\int_{\Omega} u_{j} \Delta u_{j} .
$$

Thus we see that

$$
\sum_{j=1}^{m}\left\langle R_{j} \mathbf{u}, R_{j} \mathbf{u}\right\rangle=m \int_{\Omega}(\operatorname{div} \mathbf{u})^{2}+2 \int_{\Omega}(\operatorname{div} \mathbf{u})^{2}-\int_{\Omega} \mathbf{u} \cdot \Delta \mathbf{u} .
$$

Finally, applicaton of (5.7) with $g \sim \operatorname{div} \mathbf{u},(5.9)$ becomes

$$
\sum_{j=1}^{m}\left\langle R_{j} \mathbf{u}, R_{j} \mathbf{u}\right\rangle=-\int_{\Omega} \mathbf{u} \cdot((m+2) \operatorname{grad} \operatorname{div}(\mathbf{u})+\Delta \mathbf{u}) .
$$

Equation (5.10) is equivalent to $(5.4 \mathrm{a})$.

For the $S_{j}$ we have

$$
\left\langle S_{j} \mathbf{u}, S_{j} \mathbf{u}\right\rangle=\int_{\Omega}\left\|\frac{\partial \mathbf{u}}{\partial x_{j}}\right\|^{2}=-\int_{\Omega} \mathbf{u} \cdot \frac{\partial^{2} \mathbf{u}}{\partial x_{j}^{2}} .
$$

Summing (5.11) over $j$ and using the definition of $\Delta$ yields the result (5.4b).

Lemma 4. Consider the subset $\left\{\mathbf{u} \in L^{2}(\Omega): \mathbf{u}=\mathbf{0}\right.$ on $\left.\partial \Omega\right\}=D$. Then the operators

$$
C_{j} \mathbf{u}=-x_{j} \mathbf{u} \text { for } j=1,2, \ldots, m
$$

are symmetric on $L^{2}(\Omega)$ and leave $D$ invariant. Moreover the commutators with the $R_{j}$ and $S_{j}$ of Lemma 3 obey

$$
\sum_{j=1}^{m}\left[R_{j}, C_{j}\right] \mathbf{u}=-2 \mathbf{u}
$$

and

$$
\sum_{j=1}^{m}\left[S_{j}, C_{j}\right] \mathbf{u}=-m \mathbf{u}
$$


Proof. We have $\left[R_{j}, C_{j}\right] \mathbf{u}=R_{j} C_{j} \mathbf{u}-C_{j} R_{j} \mathbf{u}$. Thus

$$
\begin{aligned}
{\left[R_{j}, C_{j}\right] \mathbf{u} } & =\operatorname{div}\left(-x_{j} \mathbf{u}\right) \operatorname{grad} x_{j}+\operatorname{grad}\left(-x_{j} u_{j}\right)+x_{j} \operatorname{div} \mathbf{u} \operatorname{grad} x_{j}+x_{j} \operatorname{grad} u_{j} \\
& =-u_{j} \operatorname{grad} x_{j}-\left(\operatorname{grad} x_{j}\right) u_{j}=-2 u_{j} \operatorname{grad} x_{j} .
\end{aligned}
$$

By summing (5.14) over $j$ we obtain (5.13a).

For the $S_{j}$ we have

$$
\left[S_{j}, C_{j}\right] \mathbf{u}=\frac{\partial}{\partial x_{j}}\left(-x_{j} \mathbf{u}\right)+x_{j} \frac{\partial u}{\partial x_{j}}=-\mathbf{u} .
$$

Summing (5.15) over $j$ yields the result (5.13b). The fact that the $C_{j}$ are symmetric on $L^{2}(\Omega)$ and leave $D$ invariant is easily seen.

Lemma 5. For the operators $L \mathbf{u}=-\Delta \mathbf{u}$ and $M \mathbf{u}=-\operatorname{grad} \operatorname{div} \mathbf{u}$ and the operators $C_{j}$ of Lemma 4 we have

$$
\left[L, C_{j}\right] \mathbf{u}=2 S_{j} \mathbf{u}
$$

and

$$
\left[M, C_{j}\right] \mathbf{u}=R_{j} \mathbf{u},
$$

where the operators $S_{j}$ and $R_{j}$ are as in Lemma 3. Moreover the $R_{j}$ and $S_{j}$ are skew-symmetric.

Proof. The $i$ th component of $\left[L, C_{j}\right] \mathbf{u}$ is given by

$$
\begin{aligned}
& -\Delta\left(-x_{j} u_{i}\right)-\left(-x_{j}\left(-\Delta u_{i}\right)\right)=\Delta\left(x_{j} u_{i}\right)-x_{j} \Delta u_{i} \\
& \quad=2 \operatorname{grad} x_{j} \cdot \operatorname{grad} u_{i}+x_{j} \Delta u_{i}-x_{j} \Delta u_{i}=2 \partial u_{i} / \partial x_{j} .
\end{aligned}
$$

Since (5.17) holds for each component of $\left[L, C_{j}\right] \mathbf{u}$ we see that $\left[L, C_{j}\right] \mathbf{u}=$ $2 \partial \mathbf{u} / \partial x_{j}=2 S_{j} \mathbf{u}$. We use (5.5) to establish (5.16b). We have

$$
\begin{aligned}
{\left[M, C_{j}\right] \mathbf{u} } & =-\operatorname{grad} \operatorname{div}\left(-x_{j} \mathbf{u}\right)-x_{j} \operatorname{grad} \operatorname{div} \mathbf{u} \\
& =\operatorname{grad}\left(\operatorname{grad} x_{j} \cdot \mathbf{u}\right)+\operatorname{grad}\left(x_{j} \operatorname{div} \mathbf{u}\right)-x_{j} \operatorname{grad} \operatorname{div} \mathbf{u} \\
& =\operatorname{grad} u_{j}+\operatorname{div} \mathbf{u} \operatorname{grad} x_{j}+x_{j} \operatorname{grad} \operatorname{div} \mathbf{u}-x_{j} \operatorname{grad} \operatorname{div} \mathbf{u} \\
& =\operatorname{div} \mathbf{u} \operatorname{grad} x_{j}+\operatorname{grad} u_{j}=R_{j} \mathbf{u} .
\end{aligned}
$$

The $R_{j}$ and $S_{j}$ are skew-symmetric because they are the commutators of symmetric operators.

Theorem 10. If $\Omega \subseteq \mathbb{R}^{m}$ is bounded, $\alpha \geq 0$ is a constant and problem (5.1) is selfadjoint in $\Omega$ with $\Lambda_{1} \leq \Lambda_{2} \leq \cdots \leq \Lambda_{n}<\Lambda_{n+1} \leq \cdots$ then the eigenvalues of (5.1) satisfy

$$
\sum_{i=1}^{n} \frac{\Lambda_{i}}{\Lambda_{n+1}-\Lambda_{i}} \geq \frac{m^{2} n}{4(m+\alpha)}
$$


Proof. We have established all that we need in Lemmas 3-5 above. All we need to do is to choose the $B_{j}, T_{j}$, and $\gamma$ in (2.1) properly. Define

$$
T_{j}=S_{j} \text { for } j=1,2, \ldots, m .
$$

Thus Lemma 3 implies that

$$
\sum_{j=1}^{m}\left\langle T_{j} \mathbf{u}, T_{j} \mathbf{u}\right\rangle=\langle-\Delta \mathbf{u}, \mathbf{u}\rangle \leq\langle A \mathbf{u}, \mathbf{u}\rangle .
$$

Next we define the $B_{j}$ by

$$
B_{j}=C_{j} \quad \text { for } j=1,2, \ldots, m .
$$

Thus using Lemma 4, (5.20), and (5.22) we obtain

$$
\sum_{j=1}^{m}\left\langle\left[T_{j}, B_{j}\right] \mathbf{u}, \mathbf{u}\right\rangle=-m\langle\mathbf{u}, \mathbf{u}\rangle .
$$

Since $\left[A, C_{j}\right]=\left[L, C_{j}\right]+\alpha\left[M, C_{j}\right]$, where $L$ and $M$ are defined in Lemma 5, we have $\left[A, C_{j}\right]=2 S_{j}+\alpha R_{j}$. Thus, since $B_{j}=C_{j}$

$$
\begin{aligned}
\sum_{j=1}^{m}\left[\left[A, B_{j}\right], B_{j}\right] \mathbf{u} & =2 \sum_{j=1}^{m}\left[S_{j}, B_{j}\right] \mathbf{u}+\alpha \sum_{j=1}^{m}\left[R_{j}, B_{j}\right] \mathbf{u} \\
& =-2(m+\alpha) \mathbf{u} .
\end{aligned}
$$

Placing $\mathbf{u}_{i}$, a normalized eigenvector of $A$ corresponding to $\Lambda_{i}$, into (5.21), (5.23), and (5.24) and then the results into (2.1) we obtain

$$
-m n \gamma+\gamma^{2} \sum_{i=1}^{n} \frac{\left\langle-\Delta \mathbf{u}_{i}, \mathbf{u}_{i}\right\rangle}{\beta-\Lambda_{i}}=-(m+\alpha) n .
$$

If $\beta$ solves $(5.25)$ then

$$
-m n \gamma+\gamma^{2} \sum_{i=1}^{n} \frac{\Lambda_{i}}{\beta-\Lambda_{i}} \geq-n(m+\alpha)
$$

by the upper bound in (5.21). Finally if we find a value for $\beta$ yielding equality in (5.26) it must be no less than the value of $\beta$ which solves (5.25).

Minimizing the left-hand side of (5.26) in $\gamma$ as before, and rearranging yields

$$
\sum_{i=1}^{n} \frac{\Lambda_{i}}{\beta-\Lambda_{i}}=\frac{m^{2} n}{4(m+\alpha)} .
$$

Equation (5.27) has a solution $\beta>\Lambda_{n}$ so we can conclude that

$$
\sum_{i=1}^{n} \frac{\Lambda_{i}}{\Lambda_{n+1}-\Lambda_{i}} \geq \frac{m^{2} n}{4(m+\alpha)},
$$

the desired result. 
An earlier result of this author [6] for problem (5.1) was obtained in explicit form

$$
\Lambda_{k} \leq\left(\frac{m+\alpha k}{m}\right)\left(\Lambda_{(j-1) m+1}+\frac{4}{m j} \sum_{i=1}^{j} \Lambda_{(i-1) m+1}\right)
$$

where $j$ is the largest integer which does not exceed $\mathrm{k} / \mathrm{m}$. It is difficult to compare result (5.19) to (5.29) in general. However for $k=m=2,(5.29)$ becomes

$$
\Lambda_{2} \leq(1+\alpha) 3 \Lambda_{1},
$$

and (5.19) can be rearranged to become

$$
\Lambda_{2} \leq\left(1+\frac{4(2+\alpha)}{4}\right) \Lambda_{1}=(3+\alpha) \Lambda_{1} .
$$

Clearly the upper bound in (5.31) is smaller than that of (5.30) in this case.

\section{REFERENCES}

1. Zu-chi Chen, Inequalities for eigenvalues of a class of polyharmonic operators, Applicable Anal. 27 (1988), 289-314.

2. G. N. Hile and M. H. Protter, Inequalities for eigenvalues of the Laplacian, Indiana Univ. Math. J. 29 (1980), 523-538.

3. G. N. Hile and R. Z. Yeh, Inequalities for eigenvalues of the biharmonic operator, Pacific J. Math. 112 (1984), 115-133.

4. S. M. Hook, Inequalities for eigenvalues of self-adjoint operators, Doctoral Dissertation, University of California, Berkeley, 1986.

5. __ Inequalities for eigenvalues of selfadjoint operators, Trans. Amer. Math. Soc. $\mathbf{3 1 8}$ (1990), 237-259.

6. __ Bounds for the fundamental frequencies of an elastic medium, Preprint.

7. B. Kawohl and G. Sweers, Remarks on eigenvalues and eigenfunctions of a special elliptic system, Z. Angew. Math. Phys. 38 (1987), 730-740.

8. L. E. Payne, G. Polya and H. F. Weinberger, On the ratio of consecutive eigenvalues, J. Math. Phys. 35 (1956), 289-298.

Department of Mathematics, University of California, Davis, California 95616

Current address: 869 Rosemount Road, Oakland, California 94610 